\title{
Adaptive Beamforming Based on Complex Quaternion Processes
}

\author{
Jian-wu Tao ${ }^{1}$ and Wen-xiu Chang ${ }^{2}$ \\ ${ }^{1}$ Department of Flight Vehicle Control, Aviation University, Changchun 130022, China \\ ${ }^{2}$ College of Communication Engineering, Jilin University, Changchun 130025, China
}

Correspondence should be addressed to Jian-wu Tao; jianwu.tao@gmail.com

Received 20 February 2014; Accepted 21 May 2014; Published 12 June 2014

Academic Editor: Chunlin Chen

Copyright (c) 2014 J.-w. Tao and W.-x. Chang. This is an open access article distributed under the Creative Commons Attribution License, which permits unrestricted use, distribution, and reproduction in any medium, provided the original work is properly cited.

\begin{abstract}
Motivated by the benefits of array signal processing in quaternion domain, we investigate the problem of adaptive beamforming based on complex quaternion processes in this paper. First, a complex quaternion least-mean squares (CQLMS) algorithm is proposed and its performance is analyzed. The CQLMS algorithm is suitable for adaptive beamforming of vector-sensor array. The weight vector update of CQLMS algorithm is derived based on the complex gradient, leading to lower computational complexity. Because the complex quaternion can exhibit the orthogonal structure of an electromagnetic vector-sensor in a natural way, a complex quaternion model in time domain is provided for a 3-component vector-sensor array. And the normalized adaptive beamformer using CQLMS is presented. Finally, simulation results are given to validate the performance of the proposed adaptive beamformer.
\end{abstract}

\section{Introduction}

As an important tool of multidimensional signal processing, the quaternion algebra has been applied to parameter estimation of two-dimensional (2D) harmonic signals [1], direction-of-arrival (DOA) estimation of polarized signals [2-4], image processing [5], space-time-polarization block codes [6], Kalman filtering [7], adaptive filtering [8, 9], independent component analysis (ICA) algorithm [10], widely linear modelling and filtering [11-13], nonlinear adaptive filtering [14], and blind source separation [15]. The potential advantages of quaternion signal processing are as follows. (1) The orthogonality between each component is naturally considered, leading to improved accuracies of signal processing. (2) Signal in different components can be represented as a single-signal, leading to reduced complexity of processing approach.

An electromagnetic (EM) vector-sensor belongs to a polarization-sensitive sensor. It can measure both horizontal and vertical polarization components of the received waveform. By employing the polarization diversity of polarized signals, the performance of beamformers can be enhanced $[16,17]$. Thus, spatiotemporal-polarization processing based on EM vector-sensor arrays had come to some researcher's notice. In [18], a minimum variance distortionless response (MVDR) beamformer with an EM vector-sensor was presented and its performance was analyzed. In [19], a blind linearly constrained minimum variance (LCMV) beamformer was investigated for multiple wideband fast frequency-hop signals. But, these researches are based on the so-called "long vector" model. In conventional "long vector" based approaches, six components of an EM vector-sensor are treated as six independent and peer-to-peer elements, so that the output data of each component is arranged in a long vector. The main advantage of "long vector" based approaches is that it can use the well-known matrix algebra over the real or the complex field. The "long vector" based approaches, however, have the drawback of destroying locally the orthogonal structure of an EM vector-sensor [2,3]. Instead of "long vector" based approach, the complex quaternion domain facilitates modelling and processing of four-dimensional complex signals. The complex quaternion model can exhibit the orthogonal structure of an EM vector-sensor in a natural way. Motivated by the benefits of array signal processing in quaternion domain, the quaternion beamformers [20-26] were recently 
developed. In [20], a quaternion minimum mean square error (MMSE) algorithm was proposed and applied to the beamforming of a two-component vector-sensor array. In [22], a quaternion-capon beamformer using a crossed dipole array was proposed to improve the robustness of Capon beamformer. In [23, 24], a quaternion MVDR beamformer with an interference and noise canceller (INC) was proposed to increase the output signal to interference-plus-noise ratio, and its performance was analyzed. In [25], a quaternion spatial matched filter (QSMF) is presented and its characteristics are analyzed. Based on quaternion widely linear processing, a generalized sidelobe canceller (GSC) was proposed in [26]. But, these beamformers are only suitable for 2-component vector-sensor array. To make use of 3-component vectorsensor array, the complex quaternions may be introduced in adaptive beamforming. The adaptive beamformers can adapt to the incoming data. They can be divided into three general categories [27]: (1) beamformers with the sample matrix inversion (SMI) or the direct matrix inversion (DMI) technique, implementation of which requires the inversion of the sample covariance matrix. This category includes the LCMVSMI and MVDR-SMI beamformers, (2) beamformers with the recursive least squares (RLS) algorithm, which implement the inversion recursively. This category includes the MVDRRLS and LCMV-GSC-RLS beamformers, and (3) beamformers with the least-mean squares (LMS) algorithm, which adapt classical steepest descent algorithms to the optimization problem in order to find the optimal weight vector. This category includes the MMSE-LMS and GSC-LMS beamformers. Unfortunately, adaptive beamforming based on complex quaternion processes has received a little attention.

In this paper, we propose a complex quaternion leastmean squares (CQLMS) algorithm and investigate its performances. The CQLMS algorithm is suitable for adaptive beamforming of vector-sensor array. Unlike the quaternion least-mean squares (QLMS) algorithm [8], the weight vector update of the CQLMS algorithm is derived based on the complex gradient. Thus, the computational complexity of the CQLMS algorithm is less than that of the QLMS algorithm. Further, a complex quaternion model in time domain is provided for a 3-component vector-sensor array. And the normalized adaptive beamformer using the proposed CQLMS algorithm is presented. From the incoming data, the weight vector of this adaptive beamformer can be adaptively updated using the proposed CQLMS algorithm.

\section{Complex Quaternion Algebra}

The complex quaternion algebra (or biquaternion algebra) is well known as a four- dimensional vector space over the complex number field $\mathbf{C}$. In the complex quaternion number field $\mathbf{Q}, 1, i, j$, and $k$ denote four basis elements and they satisfy the following multiplication laws:

$$
\begin{aligned}
& i^{2}=j^{2}=k^{2}=-1 ; \quad i j k=-1 ; \\
& i j=k=-j i ; \quad j k=i=-k j ; \\
& k i=j=-i k
\end{aligned}
$$

and 1 acts as a unity element.
2.1. Complex Quaternions. A complex quaternion variable $x \in \mathbf{Q}$ can be written in its Cartesian form as $x=x_{0}+x_{1} i+$ $x_{2} j+x_{3} k$, where $x_{0}, x_{1}, x_{2}, x_{3} \in \mathbf{C}$. According to this definition, real numbers, complex numbers, and real quaternions can be regarded as the special cases of complex quaternions.

Some known terminologies on complex quaternions are listed below. For $x=x_{0}+x_{1} i+x_{2} j+x_{3} k \in \mathbf{Q}$, it can be written as $x=\delta(x)+\mathscr{V}(x)$, where $\delta(x)=x_{0}$ is the scalar component of $x$ and $\mathscr{V}(x)=x_{1} i+x_{2} j+x_{3} k$ is the vector component of $x$. Also, $x$ can be written as $x=\mathscr{R}(x)+I \mathscr{I}(x)$, where

$$
\begin{aligned}
& \mathscr{R}(x)=\mathscr{R}\left(x_{0}\right)+\mathscr{R}\left(x_{1}\right) i+\mathscr{R}\left(x_{2}\right) j+\mathscr{R}\left(x_{3}\right) k \\
& \mathscr{I}(x)=\mathscr{I}\left(x_{0}\right)+\mathscr{I}\left(x_{1}\right) i+\mathscr{I}\left(x_{2}\right) j+\mathscr{I}\left(x_{3}\right) k
\end{aligned}
$$

are, respectively, the real and imaginary components of $x$ and $\mathscr{R}(x)$ and $\mathscr{I}(x)$ are real quaternions. The relations between complex imaginary unit $I=\sqrt{-1}$ and quaternion imaginary units are

$$
i I=I i ; \quad j I=I j ; \quad k I=I k .
$$

The complex conjugate of $x$ is defined as $x^{*}=x_{0}^{*}+x_{1}^{*} i+x_{2}^{*} j+$ $x_{3}^{*} k$, where $x_{k}^{*}(k=0,1,2,3)$ are the complex conjugate of complex coefficients of $x$. The quaternion conjugate of $x$ is defined as $x^{\star}=x_{0}-x_{1} i-x_{2} j-x_{3} k=\delta(x)-\mathscr{V}(x)$. The complex quaternion (Hermitian) conjugate of $x$ is defined as $x^{\dagger}=$ $x_{0}^{*}-x_{1}^{*} i-x_{2}^{*} j-x_{3}^{*} k=\left(x^{*}\right)^{\star}=\left(x^{\star}\right)^{*}$. The norm of $x$, noted by $|x|$, is given by

$$
\begin{aligned}
|x| & =\sqrt{\left|x_{0}\right|^{2}+\left|x_{1}\right|^{2}+\left|x_{2}\right|^{2}+\left|x_{3}\right|^{2}} \\
& =\sqrt{\mathcal{S}\left(x x^{\dagger}\right)} .
\end{aligned}
$$

Note that the complex quaternions are not a normed algebra under this norm, so in general $|x y| \neq|x||y|$ for $x, y \in \mathbf{Q}$.

Some known basic properties on complex quaternions are listed below: let $x, y \in \mathbf{Q}$ be given. Then

(1) $\left(x^{*}\right)^{*}=x,\left(x^{\star}\right)^{\star}=x,\left(x^{\dagger}\right)^{\dagger}=x$;

(2) $(x+y)^{*}=x^{*}+y^{*},(x+y)^{\star}=x^{\star}+y^{\star},(x+y)^{\dagger}=x^{\dagger}+y^{\dagger}$;

(3) $(x y)^{*}=x^{*} y^{*},(x y)^{\star}=y^{\star} x^{\star},(x y)^{\dagger}=y^{\dagger} x^{\dagger}$;

\subsection{Vectors and Matrices of Complex Quaternions}

2.2.1. Complex Quaternion-Valued Vectors. The scalar product of two complex quaternion-valued vectors $\mathbf{a}, \mathbf{b} \in \mathbf{Q}^{N}$ is defined as

$$
\langle\mathbf{a}, \mathbf{b}\rangle_{\mathbf{Q}^{N}}=\mathbf{a}^{\ddagger} \mathbf{b}=\sum_{n=1}^{N} \mathbf{a}_{n}^{\dagger} \mathbf{b}_{n},
$$

where $\ddagger$ denotes Hermitian conjugate-transposition. With this definition, the norm of a complex quaternion-valued vector $\mathbf{a} \in \mathbf{Q}^{N}$ is given by

$$
\|\mathbf{a}\|=\sqrt{\mathcal{S}\left(\mathbf{a}^{\ddagger} \mathbf{a}\right)} .
$$


2.2.2. Complex Quaternion-Valued Matrices. For any complex quaternion-valued matrix $\mathbf{A}=\left(a_{s t}\right) \in \mathbf{Q}^{M \times N}$, the quaternion conjugate-transposition of $\mathbf{A}$ is $\mathbf{A}^{\triangleleft}=\left(a_{t s}^{\star}\right) \in \mathbf{Q}^{N \times M}$; the complex quaternion conjugate-transposition of $\mathbf{A}$ is $\mathbf{A}^{\ddagger}=$ $\left(a_{t s}^{\dagger}\right) \in \mathbf{Q}^{N \times M}$. A square matrix $\mathbf{A} \in \mathbf{Q}^{M \times M}$ is said to be Hermitian if $\mathbf{A}^{\ddagger}=\mathbf{A}$ and unitary if $\mathbf{A} \mathbf{A}^{\ddagger}=\mathbf{A}^{\ddagger} \mathbf{A}=\mathbf{I}_{M}$. It is invertible if there is a matrix $\mathbf{B}$ over $\mathbf{Q}^{M \times M}$ such that $\mathbf{A B}=$ $\mathbf{B A}=\mathbf{I}_{M}$.

Several basic properties on complex quaternion-valued matrices are listed below: given two matrices $\mathbf{A} \in \mathbf{Q}^{M \times N}$ and $\mathbf{B} \in \mathbf{Q}^{N \times P}$, then

(1) $\left(\mathbf{A}^{\triangleleft}\right)^{\triangleleft}=\mathbf{A},\left(\mathbf{A}^{\ddagger}\right)^{\ddagger}=\mathbf{A}$;

(2) $(\mathbf{A B})^{\triangleleft}=\mathbf{B}^{\triangleleft} \mathbf{A}^{\triangleleft},(\mathbf{A B})^{\ddagger}=\mathbf{B}^{\ddagger} \mathbf{A}^{\ddagger}$;

(3) $(\mathbf{A B})^{-1}=\mathbf{B}^{-1} \mathbf{A}^{-1}$, if $\mathbf{A}$ and $\mathbf{B}$ are invertible;

(4) $\left(\mathbf{A}^{\triangleleft}\right)^{-1}=\left(\mathbf{A}^{-1}\right)^{\triangleleft},\left(\mathbf{A}^{\ddagger}\right)^{-1}=\left(\mathbf{A}^{-1}\right)^{\ddagger}$, if $\mathbf{A}$ is invertible.

2.2.3. Complex Representation of a Complex QuaternionValued Matrices. For any complex quaternion-valued matrix $\mathbf{A}=\mathbf{A}_{0}+\mathbf{A}_{1} i+\mathbf{A}_{2} j+\mathbf{A}_{3} k \in \mathbf{Q}^{M \times N}$, its complex representation $\Psi(\mathbf{A}) \in \mathbf{C}^{2 M \times 2 N}$ can be written as

$$
\Psi(\mathbf{A})=\left[\begin{array}{cc}
\mathbf{A}_{0}+\mathbf{A}_{1} I & -\left(\mathbf{A}_{2}+\mathbf{A}_{3} I\right) \\
\mathbf{A}_{2}-\mathbf{A}_{3} I & \mathbf{A}_{0}-\mathbf{A}_{1} I
\end{array}\right] .
$$

Various operation properties on complex representation of complex quaternion-valued matrices are listed below: given three complex quaternion-valued matrices $\mathbf{A}, \mathbf{B} \in$ $\mathbf{Q}^{M \times N}, \mathbf{D} \in \mathbf{Q}^{N \times P}$, and a complex number $\lambda \in \mathbf{C}$, then

(1) $\mathbf{A}=\mathbf{B} \Leftrightarrow \Psi(\mathbf{A})=\Psi(\mathbf{B})$;

(2) $\Psi(\mathbf{A}+\mathbf{B})=\Psi(\mathbf{A})+\Psi(\mathbf{B}), \Psi(\mathbf{A D})=\Psi(\mathbf{A}) \Psi(\mathbf{D})$, $\Psi(\lambda \mathbf{A})=\Psi(\mathbf{A} \lambda)=\lambda \Psi(\mathbf{A}) ;$

(3) $\Psi\left(\mathbf{I}_{M}\right)=\mathbf{I}_{2 M}$;

(4) $\mathbf{A}=(1 / 4) \mathbf{E}_{2 M} \Psi(\mathbf{A}) \mathbf{E}_{2 N}^{\ddagger}$, where $\mathbf{E}_{2 t}=\left[(1-I i) \mathbf{I}_{t},(j+\right.$ Ik) $\left.\mathbf{I}_{t}\right], t=M, N$;

(5) $\mathbf{A}$ is Hermitian if and only if $\Psi(\mathbf{A})$ is Hermitian over C.

The above complex quaternions and their properties are found in $[3,28,29]$.

\section{The Complex Quaternion LMS Algorithm}

3.1. The Derivation of CQLMS Algorithm. If complex quaternion-valued series $\mathbf{x}(n)$ and complex quaternion-valued series $d(n)$ denote, respectively, the output signal vector and the desired signal, we assume that $\mathbf{x}(n)$ is a random vector and employ a linear model to estimate $d(n)$. It is given by $\widehat{d}(n)=$ $\mathbf{w}^{\ddagger}(n) \mathbf{x}(n)$, where $\mathbf{w}(n)$ is the complex quaternion-valued weight vector. The estimated error is defined as

$$
e(n)=d(n)-\widehat{d}(n)=d(n)-\mathbf{w}^{\ddagger}(n) \mathbf{x}(n) .
$$

The real-valued quadratic cost function is defined as

$$
\begin{aligned}
J(n) & =|e(n)|^{2} \\
& =\left|e_{0}(n)\right|^{2}+\left|e_{1}(n)\right|^{2}+\left|e_{2}(n)\right|^{2}+\left|e_{3}(n)\right|^{2} \\
& =\mathcal{S}\left(e(n) e^{\dagger}(n)\right) .
\end{aligned}
$$

Based on the cost function, within the steepest descent optimization, the following complex quaternion gradients need to be calculated:

$$
\begin{aligned}
\nabla J_{\mathbf{w}^{\dagger}}(n)= & \nabla J_{\mathbf{w}_{0}^{*}}(n)+\nabla J_{\mathbf{w}_{1}^{*}}(n) i \\
& +\nabla J_{\mathbf{w}_{2}^{*}}(n) j+\nabla J_{\mathbf{w}_{3}^{*}}(n) k,
\end{aligned}
$$

where $\mathbf{w}(n)=\mathbf{w}_{0}(n)+\mathbf{w}_{1}(n) i+\mathbf{w}_{2}(n) j+\mathbf{w}_{3}(n) k$. Based on the operation properties of complex gradients in [30], the gradients can be expressed as (the full derivation can be found in Appendix)

$$
\nabla J_{\mathbf{w}^{\dagger}}(n)=-\mathbf{x}(n) e^{\dagger}(n) .
$$

Subsequently, the update of the adaptive weight vector can be written as

$$
\mathbf{w}(n+1)=\mathbf{w}(n)+\mu \mathbf{x}(n) e^{\dagger}(n),
$$

where $\mu$ denotes the iterative stepsize. Equation (12) refers to the update of the adaptive weight vector.

When $\mathbf{x}(n)$ and $e(n)$ belong to real quaternion domain, (12) can be rewritten as

$$
\mathbf{w}(n+1)=\mathbf{w}(n)+\mu \mathbf{x}(n) e^{\star}(n) .
$$

It is noted that the update (13) is not identical to the update of QLMS algorithm proposed in [8]. The update of QLMS is given by

$$
\begin{aligned}
\mathbf{w}(n+1)= & \mathbf{w}(n) \\
& +\mu\left(2 e(n) \mathbf{x}^{\star}(n)-\mathbf{x}^{\star}(n) e^{\star}(n)\right) .
\end{aligned}
$$

Comparing with (13), the computation of (14) increases $M$ quaternion multiplications and $2 M$ quaternion additions, where $M$ is the column dimension of $\mathbf{x}(n)$. A quaternion multiplication consists of 16 real multiplications and 12 real additions, and a quaternion addition consists of 4 real additions. At an update, thus, the CQLMS algorithm decreases $16 M$ real multiplications and $20 M$ real additions than the QLMS algorithm.

Further, when the complex quaternion-valued series $\mathbf{x}(n)$ and $d(n)$ degenerate to the complex-valued series, (12) can be rewritten as

$$
\mathbf{w}(n+1)=\mathbf{w}(n)+\mu \mathbf{x}(n) e^{*}(n) .
$$

Equation (15) is identical to the update of the adaptive weight vector for complex LMS (CLMS) algorithm [31]. Thus, the CLMS algorithm can be regarded as the special case of CQLMS algorithm. 
3.2. The Property of CQLMS Algorithm. To analyze the convergence of CQLMS algorithm in the mean, we assume that $d(n)=\mathbf{w}_{\text {opt }}^{\ddagger} \mathbf{x}(n)$, where $\mathbf{w}_{\text {opt }}$ is the optimal weight vector. Defining the weight error vector as $\mathbf{V}(n)=\mathbf{w}(n)-\mathbf{w}_{\text {opt }}$, then the error $e(n)$ is given by

$$
\begin{aligned}
e(n) & =d(n)-y(n) \\
& =\mathbf{w}_{\mathrm{opt}}^{\ddagger} \mathbf{x}(n)-\mathbf{w}^{\ddagger}(n) \mathbf{x}(n) \\
& =-\mathbf{V}^{\ddagger}(n) \mathbf{x}(n) .
\end{aligned}
$$

Inserting (16) into (12) and subtracting $\mathbf{w}_{\text {opt }}$ from both sides of (12), we have

$$
\begin{aligned}
\mathbf{V}(n+1) & =\mathbf{V}(n)-\mu \mathbf{x}(n) \mathbf{x}^{\ddagger}(n) \mathbf{V}(n) \\
& =\left[\mathbf{I}-\mu \mathbf{R}_{x x}(n)\right] \mathbf{V}(n),
\end{aligned}
$$

where $\mathbf{R}_{x x}(n)=\mathbf{x}(n) \mathbf{x}^{\ddagger}(n)$. Based on the properties on complex representation of complex quaternion-valued matrices, the complex representation of (17) can be written as

$$
\Psi(\mathbf{V}(n+1))=\left[\Psi(\mathbf{I})-\mu \Psi\left(\mathbf{R}_{x x}(n)\right)\right] \Psi(\mathbf{V}(n))
$$

Since $\mathbf{R}_{x x}(n)$ is a complex quaternion Hermitian matrix, $\Psi\left(\mathbf{R}_{x x}(n)\right)$ and $E\left\{\Psi\left(\mathbf{R}_{x x}(n)\right)\right\}$ are complex Hermitian matrix, where $E\{\cdot\}$ denotes the mathematical expectation operation. Then the maximum eigenvalue $\lambda_{\max }$ of complex matrix $E\left\{\Psi\left(\mathbf{R}_{x x}(n)\right)\right\}$ is also real, since the eigenvalues of complex Hermitian matrix are real. According to the convergent analysis of CLMS in [31], we have the following consequence: if the stepsize $\mu$ satisfies $0<\mu<2 / \lambda_{\max }$, the complex matrix $\Psi(\mathbf{V}(n))$ converges to zero along with an increase of iterative number $n$. Because $\mathbf{V}(n)=(1 / 4) \mathbf{E}_{2} \Psi(\mathbf{V}(n)) \mathbf{E}_{2 M}^{\ddagger}$, where $M$ denotes the dimension of the column vector $\mathbf{V}(n)$, the convergence of $\Psi(\mathbf{V}(n))$ results in the convergence of $\mathbf{V}(n)$. We come to the conclusion that if $0<\mu<2 / \lambda_{\text {max }}, \mathbf{V}(n)$ converges to zero along with an increase of iterative number $n$. The convergence of weight error vector $\mathrm{V}(n)$ indicates that the weight vector $\mathbf{w}(n)$ tends to $\mathbf{w}_{\mathrm{opt}}$ along with an increase of iterative number $n$.

\section{The Beamforming of Vector-Sensor Array Using CQLMS Algorithm}

In this section, we apply CQLMS algorithm to adaptive beamforming of vector-sensor array. First, the complex (or real) quaternion model of vector-sensor is introduced. This quaternion model is different from the quaternion model proposed in $[2,3]$. The quaternion model proposed in this paper is a time domain model of output data, whereas the quaternion model proposed in $[2,3]$ is a frequency-domain model of output data. Second, the normalized adaptive beamformer using CQLMS is presented.

4.1. Complex Quaternion Model of 3-Component VectorSensor. Consider a scenario with a vector-sensor mounted on an aircraft, assuming that the Cartesian coordinates of

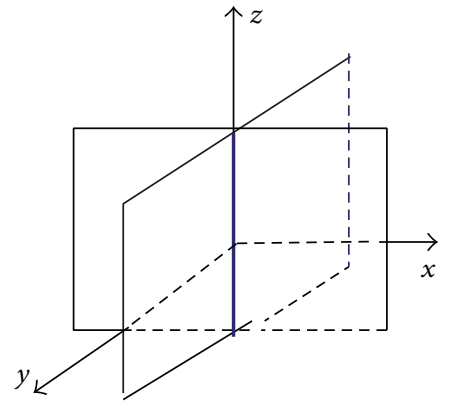

(a) A 3-component vector-sensor (b) A 2-component vector-sensor

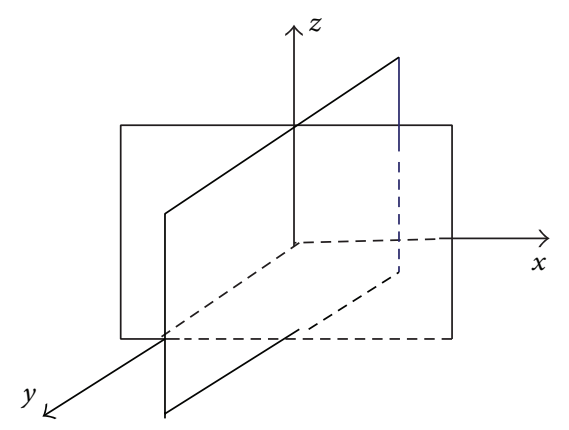

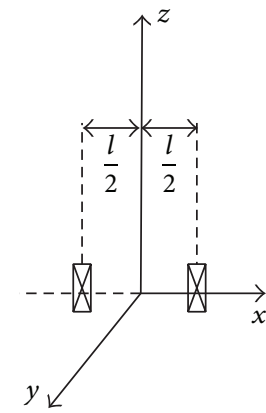

(c) A linear uniform array
FIGURE 1: A linear uniform array with two 3-component (or 2-component) vector-sensors.

vector-sensor are consistent with the airframe coordinates; that is, the airframe locates on $x$ - $y$ plane and the $z$-axis points into the airframe. The airframe may form a reflection plane when the wavelength of an incidence EM-wave is less than the size of airframe. The studies in $[32,33]$ had indicated that the mutual coupling between two collocated orthogonally oriented magnetic loops, which are along $x$ - and $y$-axis, and airframes is minimum. Thus, we construct a 3-component vector-sensor by using two collocated orthogonally oriented magnetic loops along $x$ - and $y$-axis and one dipole along $z$; see Figure 1(a). In the Cartesian coordinates, the 3-component vector-sensor has the following responses (ignoring a common constant that is determined by the antenna parameters and the distance to the antenna) [34]:

$$
\mathbf{V}_{3}(\theta, \phi)=\left[\begin{array}{l}
h_{x} \\
h_{y} \\
e_{z}
\end{array}\right]=\left[\begin{array}{cc}
-\cos \phi \sin \theta & \sin \phi \\
-\sin \phi \sin \theta & -\cos \phi \\
0 & \cos \theta
\end{array}\right]
$$

where $-\pi / 2 \leq \theta \leq \pi / 2$ is the elevation angle of incidence EM-wave and $-\pi \leq \phi<\pi$ is the azimuth angle of incidence EM-wave.

Consider a narrowband, transverse electromagnetic plane-wave traveling in an isotropic, homogeneous medium; we assume that its electric field can be represented by

$$
\boldsymbol{\xi}(t)=\left[\begin{array}{l}
\xi_{h}(t) \\
\xi_{v}(t)
\end{array}\right]=\left[\begin{array}{l}
a_{h}(t) e^{I \varphi_{h}(t)} \\
a_{v}(t) e^{I \varphi_{v}(t)}
\end{array}\right] s(t),
$$


where $a_{h}(t)$ and $a_{v}(t)$ are the time-variant amplitudes of two orthogonal components in the electric field and $\varphi_{h}(t)$ and $\varphi_{v}(t)$ are the time-variant phases of two orthogonal components. We assume that $a_{h}(t), a_{v}(t), \varphi_{h}(t)$, and $\varphi_{v}(t)$ are a stationary stochastic process; $a_{v}(t) / a_{h}(t)=\tan \gamma=\sin \gamma / \cos \gamma$ and $\varphi_{\nu}(t)-\varphi_{h}(t)=\eta$, where $\gamma$ and $\eta$ are constants. In this scenario, the electromagnetic wave is a completely polarized wave and the waveform polarization can be described by the so-called polarization ellipse. Thus $0 \leq \gamma<\pi / 2$ represents the auxiliary polarization angle, and $-\pi \leq \eta<\pi$ signifies the polarization phase difference. $s(t)$ is the scalar complex envelope of the waveform.

When a narrowband plane-wave impinges on 3-component vector-sensor from direction $(\theta, \phi)$, three temporal signals $x_{x}(t), x_{y}(t)$, and $x_{z}(t)$ are recorded on magnetic loop antennae in $x$ - and $y$-axis and dipole antenna in $z$, respectively. They are given by

$$
\begin{aligned}
{\left[\begin{array}{l}
x_{x}(t) \\
x_{y}(t) \\
x_{z}(t)
\end{array}\right] } & =\mathbf{V}_{3}(\theta, \phi)\left[\begin{array}{c}
\xi_{h}(t) \\
\xi_{v}(t)
\end{array}\right] \\
& =\mathbf{V}_{3}(\theta, \phi)\left[\begin{array}{c}
a_{h}(t) e^{I \varphi_{h}(t)} \\
a_{v}(t) e^{I \varphi_{v}(t)}
\end{array}\right] s(t) \\
& =\mathbf{V}_{3}(\theta, \phi)\left[\begin{array}{c}
\cos \gamma \\
\sin \gamma e^{I \eta}
\end{array}\right] \underbrace{\underbrace{I \varphi_{h}(t)} s(t)}_{\stackrel{\text { def }}{=} \bar{s}(t)},
\end{aligned}
$$

where $\bar{s}(t)$ is a complex stationary stochastic process.

Using $x_{x}(t), x_{y}(t)$, and $x_{z}(t)$, the output of 3-component vector-sensor can be compactly written in terms of complex quaternions

$$
\begin{aligned}
x(t) & =x_{x}(t) i+x_{y}(t) j+x_{z}(t) k \\
& =P(\theta, \phi, \gamma, \eta) \bar{s}(t),
\end{aligned}
$$

where $i, j$, and $k$ are an imaginary unit in complex quaternions. $P(\theta, \phi, \gamma, \eta)$ is a complex quaternion; that is

$$
\begin{aligned}
P(\theta, \phi, \gamma, \eta)= & \left(-\cos \phi \sin \theta \cos \gamma+\sin \phi \sin \gamma e^{I \eta}\right) i \\
& +\left(-\sin \phi \sin \theta \cos \gamma-\cos \phi \sin \gamma e^{I \eta}\right) j \\
& +\left(\cos \theta \sin \gamma e^{I \eta}\right) k .
\end{aligned}
$$

In this way, we define a new transformation to map a threedimensional complex-valued signal space to a complex quaternion-valued signal space. Unlike [3], this new transformation does not need the Fourier transform on three outputs of sensor. Thus, this transformation is implemented more easily.

When a complex quaternion-valued added noise is considered, the complex quaternion-valued output of 3-component vector-sensor is given by

$$
x(t)=P(\theta, \phi, \gamma, \eta) \bar{s}(t)+n(t),
$$

where $n(t)=n_{x}(t) i+n_{y}(t) j+n_{z}(t) k . n_{x}(t), n_{y}(t)$, and $n_{z}(t)$ are the complex-valued added noises recorded on $x, y$, and $z$ components of the 3-component vector-sensor, respectively, where $n_{x}(t), n_{y}(t)$, and $n_{z}(t)$ are assumed to be Gaussian noise with identical variance, and they are uncorrelated.

4.2. Real Quaternion Model of 2-Component Vector-Sensor. Using only two collocated orthogonally oriented magnetic loops along $x$ - and $y$-axis, we can also construct a 2 -component vector-sensor; see Figure 1(b). When a narrowband plane-wave impinges on 2-component vector-sensor, we have

$$
\left[\begin{array}{l}
x_{x}(t) \\
x_{y}(t)
\end{array}\right]=\mathbf{V}_{2}(\theta, \phi)\left[\begin{array}{c}
\cos \gamma \\
\sin \gamma e^{i \eta}
\end{array}\right] \bar{s}(t),
$$

where

$$
\mathbf{V}_{2}(\theta, \phi) \stackrel{\text { def }}{=}\left[\begin{array}{l}
h_{x} \\
h_{y}
\end{array}\right]=\left[\begin{array}{cc}
-\cos \phi \sin \theta & \sin \phi \\
-\sin \phi \sin \theta & -\cos \phi
\end{array}\right]
$$

is the responses of 2-component vector-sensor.

Since a quaternion may be expressed by two complex numbers, the output model of 2-component vector-sensor can be compactly written in terms of quaternion:

$$
x(t)=x_{x}(t)+j x_{y}(t)=P(\theta, \phi, \gamma, \eta) \bar{s}(t),
$$

where $P(\theta, \phi, \gamma, \eta)$ is a quaternion; that is,

$$
\begin{aligned}
P(\theta, \phi, \gamma, \eta)= & -\cos \phi \sin \theta \cos \gamma+\sin \phi \sin \gamma e^{i \eta} \\
& +j\left(-\sin \phi \sin \theta \cos \gamma-\cos \phi \sin \gamma e^{i \eta}\right) .
\end{aligned}
$$

In this way, we define a transformation to map a two-dimensional complex-valued signal space to a quaternion-valued signal space.

When the quaternion-valued added noise is considered, the quaternion-valued output of 2-component vector-sensor is given by

$$
x(t)=P(\theta, \phi, \gamma, \eta) \bar{s}(t)+n(t),
$$

where $n(t)=n_{x}(t)+j n_{y}(t)$.

4.3. The Beamforming of Vector-Sensor Array. Consider that a three-dimensional array consists of $M$ arbitrarily spaced but identically oriented 3-component (or 2-component) vectorsensors. In this three-dimensional array, the $m$ th vector-sensor has the location $\mathbf{r}_{m}=\left[x_{m}, y_{m}, z_{m}\right]^{T}(1 \leq m \leq M)$. Taking the first vector-sensor as reference, the inter-vector-sensor spatial phase-factor vector of array is

$$
\mathbf{q}(\theta, \phi)=\left[1, e^{I \psi_{2}}, \ldots, e^{I \psi_{M}}\right]
$$

where $\psi_{m}=(2 \pi / \lambda) \mathbf{u}^{T} \mathbf{r}_{m} \cdot \mathbf{u}=[\sin \theta \cos \phi, \sin \theta \sin \phi, \cos \theta]^{T}$ denotes direction-cosine vector and $\lambda$ is the wavelength of incidence EM-waves.

Assume a scenario of $K$ incoherent and complete polarization waves with $2 \mathrm{D}$ direction angles $\left(\theta_{k}, \phi_{k}\right)$ and polarization parameters $\left(\gamma_{k}, \eta_{k}\right)$ impinging on this three-dimensional 


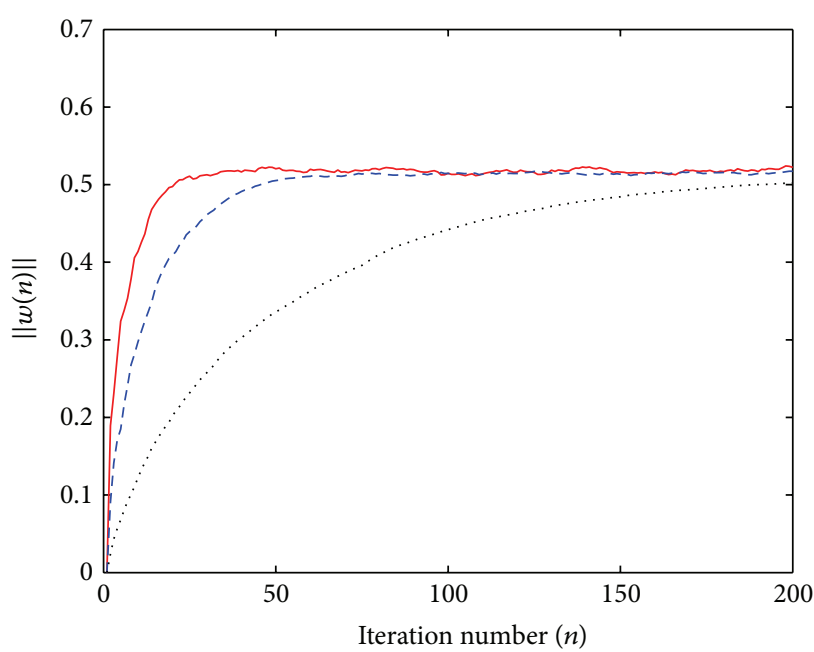

$-\mu=0.8, \beta=0.9$

$--\mu=0.4, \beta=0.9$

(a) The norm of weight vector versus iteration number $n$

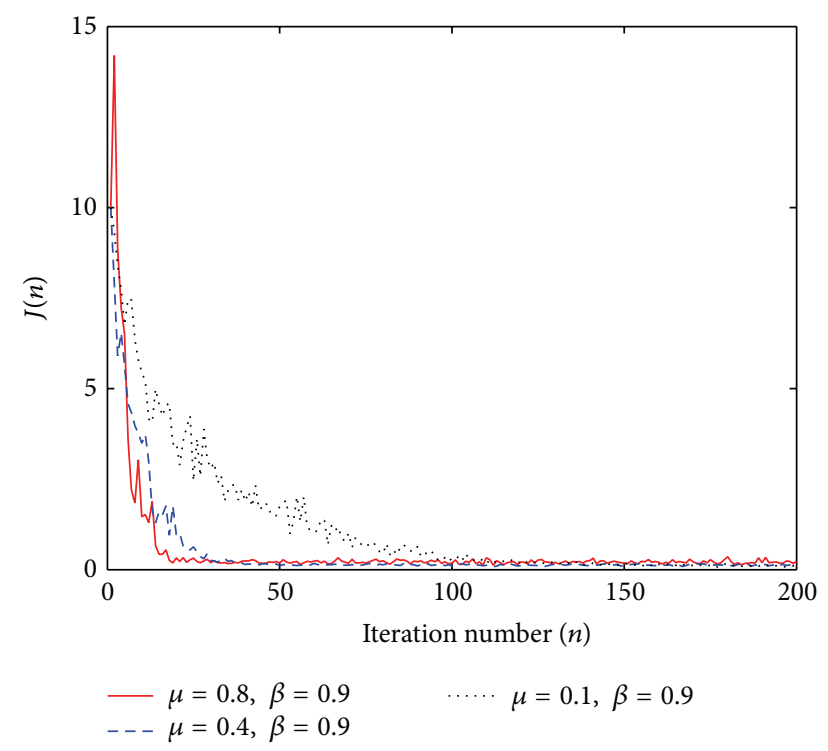

(b) The cost function $J(n)$ versus iteration number $n$

FIGURE 2: The performance of the CQLMS beamformer for $\beta=0.9$ and $\mu=0.1, \mu=0.4, \mu=0.8$.

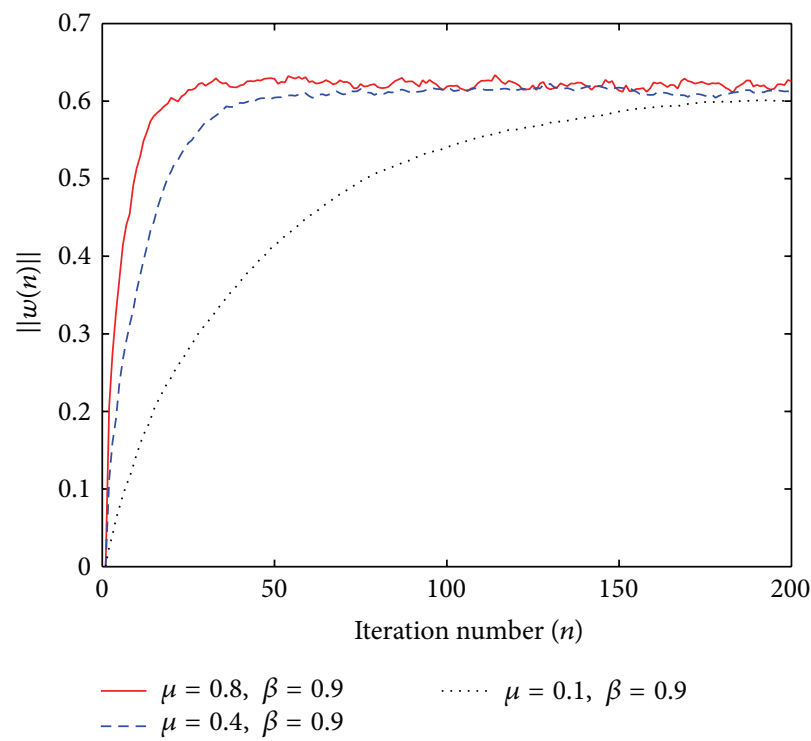

(a) The norm of weight vector versus iteration number $n$

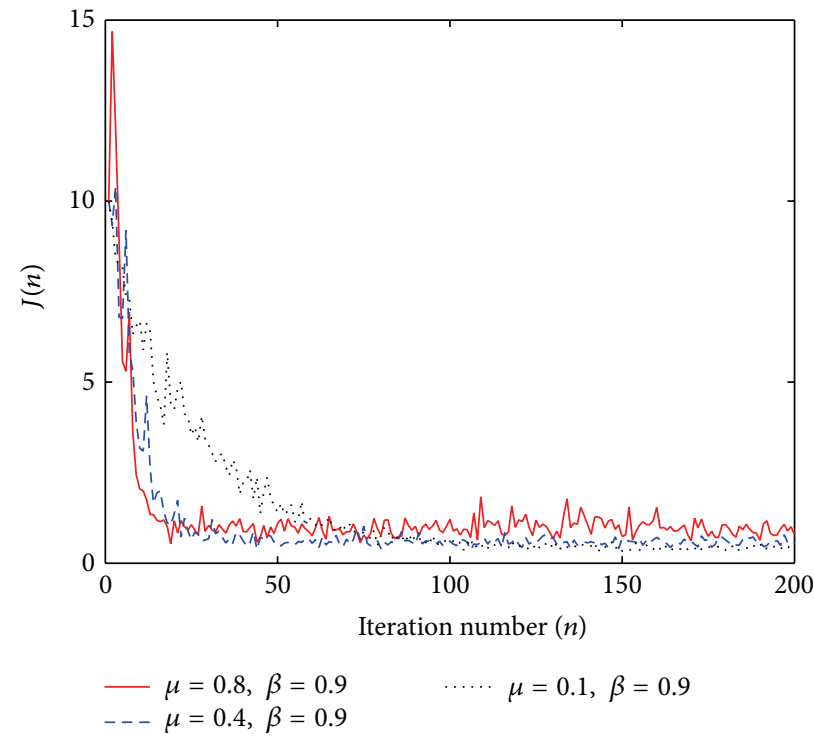

(b) The cost function $J(n)$ versus iteration number $n$

FIgURE 3: The performance of the CLMS beamformer for $\beta=0.9$ and $\mu=0.1, \mu=0.4, \mu=0.8$.

array; the output of array is given by a column vector $\mathbf{x}(t) \epsilon$ $\mathbf{Q}^{M}$ :

$$
\begin{aligned}
\mathbf{x}(t) & =\sum_{k=1}^{K} P\left(\theta_{k}, \phi_{k}, \gamma_{k}, \eta_{k}\right) \mathbf{q}\left(\theta_{k}, \phi_{k}\right) \bar{s}_{k}(t)+\mathbf{N}(t) \\
& =\sum_{k=1}^{K} \mathbf{a}_{k} \bar{s}_{k}(t)+\mathbf{N}(t),
\end{aligned}
$$

where $\mathbf{a}_{k}=P\left(\theta_{k}, \phi_{k}, \gamma_{k}, \eta_{k}\right) \mathbf{q}\left(\theta_{k}, \phi_{k}\right)$ denotes the steering vector.
The series $\mathbf{x}(n)$ obtained by sampling $\mathbf{x}(t)$ is multiplied by a weight vector to produce the array output series; it is given by

$$
y(n)=\mathbf{w}^{H}(n) \mathbf{x}(n) .
$$

When all of vector-sensors in array are the 3-component vector-sensor, a complex quaternion-valued output model (24) can be employed. Thus, the output series $\mathbf{x}(n)$ and weight vector series $\mathbf{w}(n)$ are the complex quaternion-valued vectors. Using the CQLMS algorithm, the update of the adaptive 


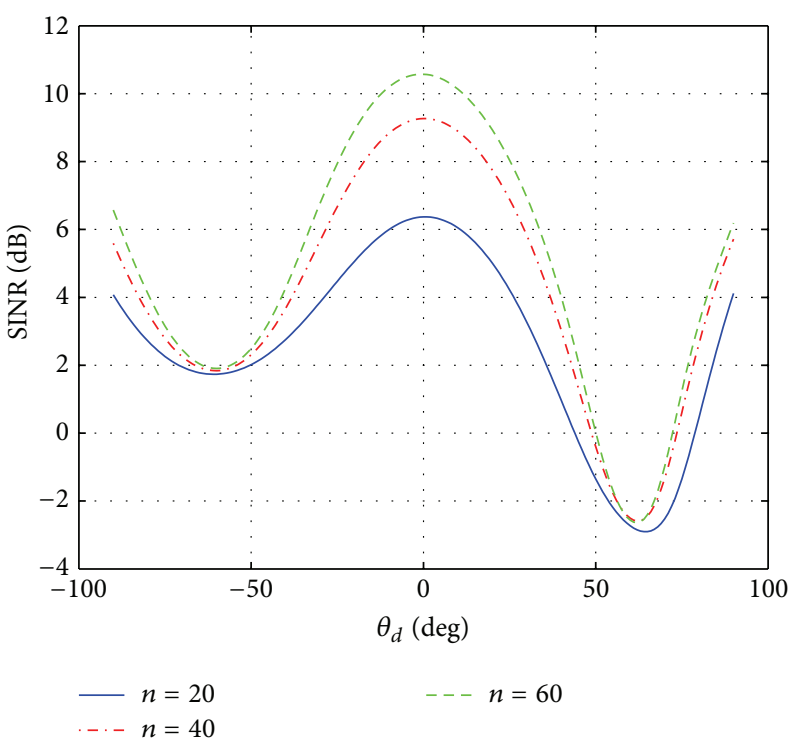

(a) Beamformer with CQLMS $(\mu=0.4, \beta=0.9)$

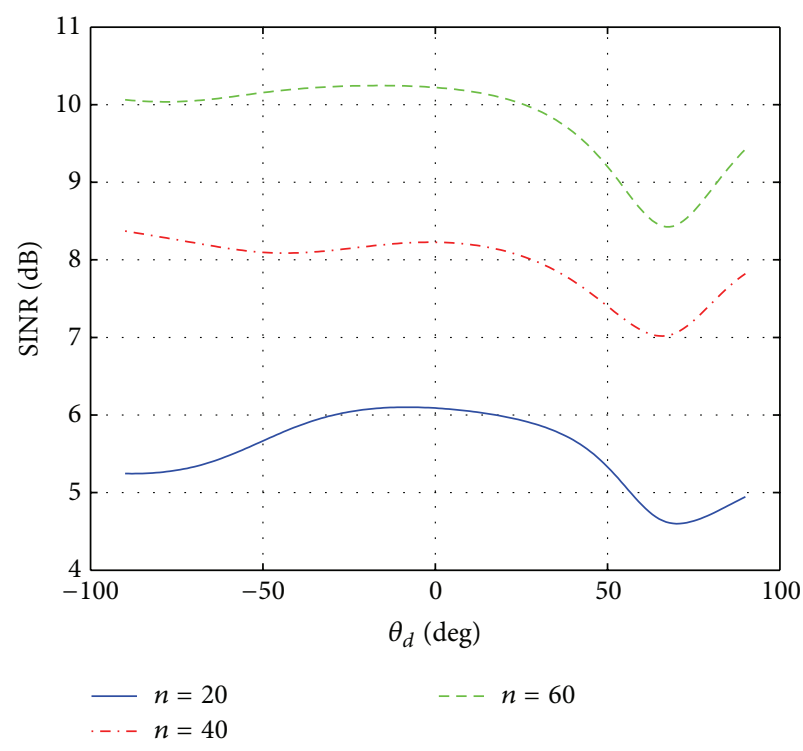

(b) MVDR-SMI beamformer

FIGURE 4: The output SINR of two beamformers versus $\theta_{d}$.

weight vector can be given by (12). In order to speed the convergence and reduce the MSE of estimates, the normalized CQLMS algorithm is adopted. Thus, the update of the adaptive weight vector becomes

$$
\mathbf{w}(n+1)=\mathbf{w}(n)+\alpha(n) \mathbf{x}(n) e^{\dagger}(n),
$$

where $\alpha(n)$ is the time-variant stepsize. In most cases, a sample-dependent stepsize is adopted [27]; that is,

$$
\alpha(n)=\frac{\mu}{\beta+\mathbf{x}^{\ddagger}(n) \mathbf{x}(n)}
$$

with $\beta>0$ and $0<\mu<1$. Note that $\beta$ is a weight value. It can avoid the fact that $\alpha(n)$ becomes too large when $\mathbf{x}^{\ddagger}(n) \mathbf{x}(n)$ is too small. Normally, $\beta$ will be close to one. The constant $\mu$ can be selected in advance. If $\mu$ is too small, the stepsize leads to slow convergence. If $\mu$ is too large, the algorithm has a large excess error or has stability problems.

When all of vector-sensors in array are the 2-component vector-sensor, a quaternion-valued output model (29) can be used. Thus, the output series $\mathbf{x}(n)$ and weight vector series $\mathbf{w}(n)$ are the real quaternion-valued vectors. Thus, the update of the adaptive weight vector is given by (13).

\section{Monte Carlo Simulations}

In this section, we evaluate the performance of the proposed algorithm by two experiments. Consider a linear array consisting of two vector-sensors; see Figure 1(c). Let $l=\lambda / 2$. Assume that two narrowband, completely polarized planewaves impinge on this array. One is the desired signal characterized by arrival angles $\left(\theta_{d}, \phi_{d}\right)$ and polarization parameters $\left(\gamma_{d}, \eta_{d}\right)$, whereas the other is the interference characterized by $\left(\theta_{i}, \phi_{i}\right)$ and $\left(\gamma_{i}, \eta_{i}\right)$. The desired signal is uncorrelated with the interference, and the interference is also uncorrelated with added noise. We define, respectively, the signal to noise ratio SNR $=10 \log \left(P_{d} / P_{n}\right)$, the interference to noise ratio INR = $10 \log \left(P_{i} / P_{n}\right)$, and the signal to interference-plus-noise ratio SINR $=10 \log \left(P_{d} /\left(P_{i}+P_{n}\right)\right)$, where $P_{d}, P_{i}$, and $P_{n}$ are the power of desired signal, the power of interference, and the power of added noise, respectively. All simulation results are based on 50 times Monte Carlo runs.

Experiment 1 (beamforming using a 3-component vector-sensor array). In this experiment, two 3-component vectorsensors are adopted in this linear array. Assume that $\theta_{d}=45^{\circ}$, $\phi_{d}=45^{\circ}, \gamma_{d}=60^{\circ}, \eta_{d}=60^{\circ}, \theta_{i}=60^{\circ}, \phi_{i}=45^{\circ}, \gamma_{i}=$ $30^{\circ}, \eta_{i}=60^{\circ}, \mathrm{SNR}=10 \mathrm{~dB}$, and INR $=20 \mathrm{~dB}$. Employing the CQLMS algorithm, Figure 2 shows the norm of weight vector $\|\mathbf{w}(n)\|$ and the cost function $J(n)$ (i.e., MSE) as a function of the iteration number $n$, with $\beta=0.9$ and various $\mu$. To compare, Figure 3 shows the simulation results of the complex LMS (CLMS) algorithm based on the "long vector" model in [16]. From Figure 2, it is shown that the CQLMS algorithm is convergent. When $\mu$ increases, the convergence is speeded up. For example, 20 iteration steps are only requested to reach stable value at $\mu=0.8$. After reaching stability, the average MSE of CQLMS algorithm is 0.15 . The convergent speed of CLMS algorithm is identical to the CQLMS algorithm, but its average MSE is 0.6. Thus, the excess error of the CLMS algorithm is larger than that of the CQLMS algorithm. Further, we compare the performance of the proposed beamformer with the MVDR-SMI beamformer in [18]. Figure 4 shows the output SINR of two beamformers versus $\theta_{d}$, where the adaptive beamformer with CQLMS algorithm ( $\mu=0.4$ and $\beta=0.9$ ) is shown in Figure 4(a) and the MVDRSMI beamformer is shown in Figure 4(b). From these curves, it is seen that the output SINR increases along with an increase of the iterative number (or snapshots) $n$ and 


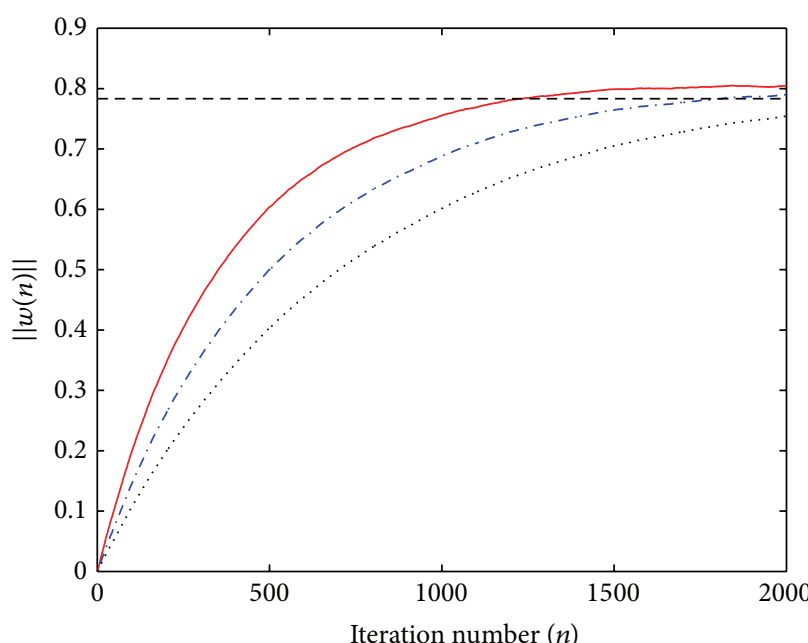

$-\mu=0.01, \beta=0.9$

-. - $\mu=0.007, \beta=0.9$

… $\mu=0.005, \beta=0.9$

- - QMMSE

(a) The norm of weight vector versus iteration number $n$

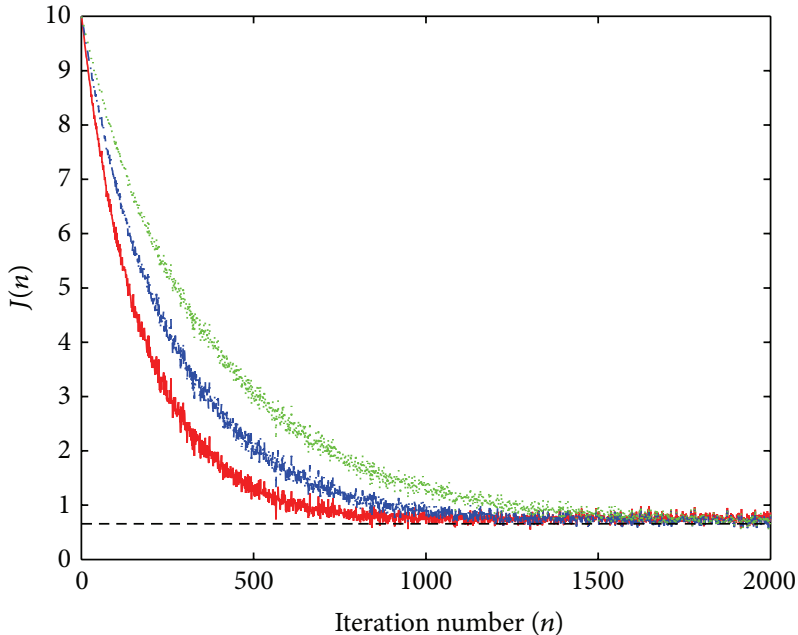

$-\mu=0.01, \beta=0.9$

- - $-\mu=0.007, \beta=0.9$

$\mu=0.005, \beta=0.9$

(b) The cost function $J(n)$ versus iteration number $n$

FIGURE 5: The performance of the CQLMS beamformer for $\beta=0.9$ and $\mu=0.01, \mu=0.007, \mu=0.005$.

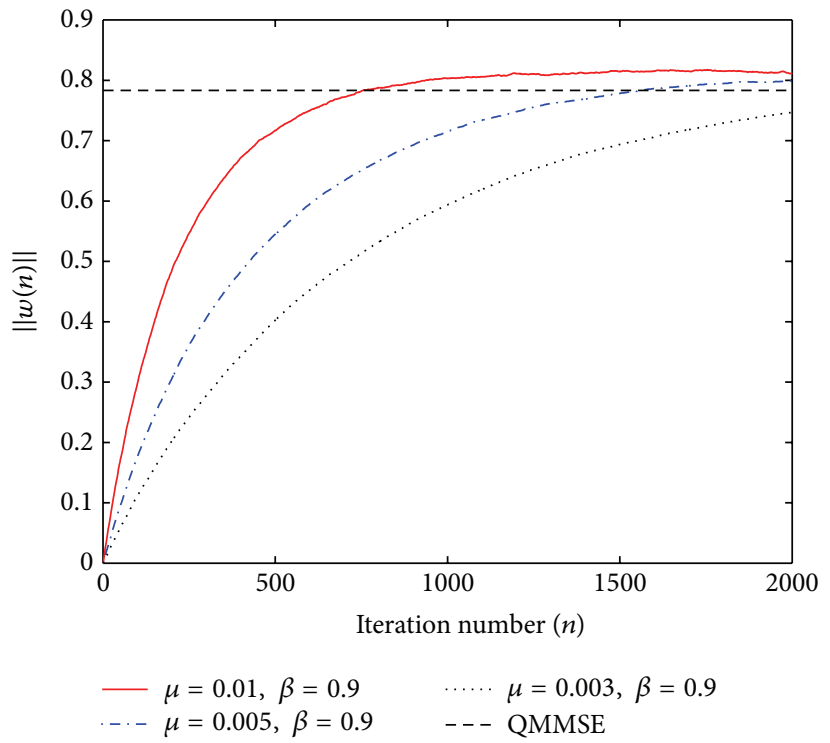

(a) The norm of weight vector versus iteration number $n$

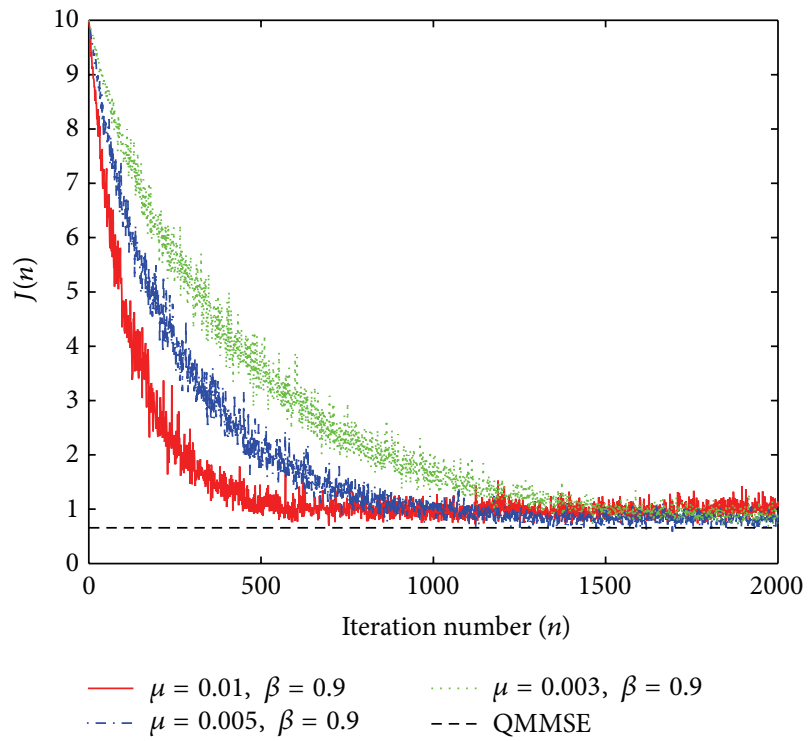

(b) The cost function $J(n)$ versus iteration number $n$

FIGURE 6: The performance of the QLMS beamformer for $\beta=0.9$ and $\mu=0.01, \mu=0.005, \mu=0.003$.

the worst output SINR is obtained if $\theta_{d}$ is close to $\theta_{i}$. This implies that when the interference is nullified, the desired signal is also nullified. When $\theta_{d}$ deviates from $\theta_{i}$, the output SINR increases. The maximum output SINR approaches up to $10 \mathrm{~dB}$. Compared with the proposed beamformer, the output SINR of the MVDR-SMI beamformer is larger when $\theta_{d}$ is close to $\theta_{i}$. This implies that the interference is not nullified effectively. In other words, the performance of the beamformer with the CQLMS algorithm is superior to that of the MVDR-SMI beamformer.
Experiment 2 (beamforming using a 2-component vector-sensor array). In this experiment, two 2-component vector-sensors are adopted in this linear array. Assume that $\theta_{d}=$ $-45^{\circ}, \phi_{d}=45^{\circ}, \gamma_{d}=45^{\circ}, \eta_{d}=60^{\circ}, \theta_{i}=60^{\circ}, \phi_{i}=45^{\circ}$, $\gamma_{i}=30^{\circ}, \eta_{i}=60^{\circ}$, SNR $=10 \mathrm{~dB}$, and INR $=20 \mathrm{~dB}$. Employing the CQLMS algorithm, Figure 5 shows the norm of weight vector $\|\mathbf{w}(n)\|$ and the cost function $J(n)$ (i.e., MSE) as a function of the iteration number $n$, with $\beta=0.9$ and various $\mu$. To compare, Figure 6 shows the simulation results of the QLMS algorithm in [8]. In Figures 5 and 6, a quaternion 
Wiener optimal solution in [20] is included, which is referred to as "QMMSE." From Figure 5, it is shown that the CQLMS algorithm is convergent. When $\mu$ increases, the convergence is speeded up. But the stable value of weight vector deviates from the optimal weight vector. For example, it is shown in Figure 5(a) that the deviation of weight vector at $\mu=0.01$ is larger than that at $\mu=0.007$. The increase of deviation results in the reduction of output SINR. After sufficient iteration steps, all curves in Figure 5(b) match the minimum MSE. Compared with the CQLMS algorithm, the excess error of the QLMS algorithm does not match the minimum MSE, as displayed in Figure 6(b). But, the QLMS algorithm may converge faster than the CQLMS algorithm.

\section{Conclusion}

In this paper, we propose a complex quaternion LMS algorithm and investigate its performance. The CQLMS algorithm is suitable for adaptive beamforming of vector-sensor array. Further, a complex quaternion model in time domain is provided for 3-component vector-sensor array. The normalized adaptive beamformer using CQLMS algorithm is presented. Simulation results show that the performances of the CQLMS algorithm are superior to that of the CLMS and QLMS algorithms. And the CQLMS algorithm is computationally less than the QLMS algorithm.

\section{Appendix}

\section{A. Derivation of the Stochastic Gradient within CQLMS}

To simplify the notation, we will ignore the time index " $(n)$ " in the following derivation. To calculate the derivatives of the error $e$ and its complex quaternion conjugate $e^{\dagger}$ with respect to the complex quaternion conjugate of weight vector $\mathbf{w}^{\dagger}$, the terms $\mathbf{w}^{\ddagger} \mathbf{x}$ and $\mathbf{x}^{\ddagger} \mathbf{w}$ can be expanded as

$$
\begin{array}{r}
\mathbf{w}^{\ddagger} \mathbf{x}=\left[\begin{array}{l}
\mathbf{w}_{0}^{H} \mathbf{x}_{0}+\mathbf{w}_{1}^{H} \mathbf{x}_{1}+\mathbf{w}_{2}^{H} \mathbf{x}_{2}+\mathbf{w}_{3}^{H} \mathbf{x}_{3} \\
\mathbf{w}_{0}^{H} \mathbf{x}_{1}-\mathbf{w}_{1}^{H} \mathbf{x}_{0}-\mathbf{w}_{2}^{H} \mathbf{x}_{3}+\mathbf{w}_{3}^{H} \mathbf{x}_{2} \\
\mathbf{w}_{0}^{H} \mathbf{x}_{2}+\mathbf{w}_{1}^{H} \mathbf{x}_{3}-\mathbf{w}_{2}^{H} \mathbf{x}_{0}-\mathbf{w}_{3}^{H} \mathbf{x}_{1} \\
\mathbf{w}_{0}^{H} \mathbf{x}_{3}-\mathbf{w}_{1}^{H} \mathbf{x}_{2}+\mathbf{w}_{2}^{H} \mathbf{x}_{1}-\mathbf{w}_{3}^{H} \mathbf{x}_{0}
\end{array}\right] \\
\mathbf{x}^{\ddagger} \mathbf{w}=\left[\begin{array}{l}
\mathbf{x}_{0}^{H} \mathbf{w}_{0}+\mathbf{x}_{1}^{H} \mathbf{w}_{1}+\mathbf{x}_{2}^{H} \mathbf{w}_{2}+\mathbf{x}_{3}^{H} \mathbf{w}_{3} \\
\mathbf{x}_{0}^{H} \mathbf{w}_{1}-\mathbf{x}_{1}^{H} \mathbf{w}_{0}-\mathbf{x}_{2}^{H} \mathbf{w}_{3}+\mathbf{x}_{3}^{H} \mathbf{w}_{2} \\
\mathbf{x}_{0}^{H} \mathbf{w}_{2}+\mathbf{x}_{1}^{H} \mathbf{w}_{3}-\mathbf{x}_{2}^{H} \mathbf{w}_{0}-\mathbf{x}_{3}^{H} \mathbf{w}_{1} \\
\mathbf{x}_{0}^{H} \mathbf{w}_{3}-\mathbf{x}_{1}^{H} \mathbf{w}_{2}+\mathbf{x}_{2}^{H} \mathbf{w}_{1}-\mathbf{x}_{3}^{H} \mathbf{w}_{0}
\end{array}\right] .
\end{array}
$$

According to the operation properties of complex gradients in [30], the derivatives of the cost function with respect to the four components of $\mathbf{w}^{\dagger}$ can be calculated from (A.1):

$$
\begin{aligned}
\nabla J_{\mathbf{w}_{0}^{*}}= & \nabla_{\mathbf{w}_{0}^{*}}\left(e_{0} e_{0}^{*}\right)+\nabla_{\mathbf{w}_{0}^{*}}\left(e_{1} e_{1}^{*}\right) \\
& +\nabla_{\mathbf{w}_{0}^{*}}\left(e_{2} e_{2}^{*}\right)+\nabla_{\mathbf{w}_{0}^{*}}\left(e_{3} e_{3}^{*}\right) \\
= & -\mathbf{x}_{0} e_{0}^{*}-\mathbf{x}_{1} e_{1}^{*}-\mathbf{x}_{2} e_{2}^{*}-\mathbf{x}_{3} e_{3}^{*}
\end{aligned}
$$

$$
\begin{aligned}
\nabla J_{\mathbf{w}_{1}^{*}}= & \nabla_{\mathbf{w}_{1}^{*}}\left(e_{0} e_{0}^{*}\right)+\nabla_{\mathbf{w}_{1}^{*}}\left(e_{1} e_{1}^{*}\right) \\
& +\nabla_{\mathbf{w}_{1}^{*}}\left(e_{2} e_{2}^{*}\right)+\nabla_{\mathbf{w}_{1}^{*}}\left(e_{3} e_{3}^{*}\right) \\
= & \mathbf{x}_{0} e_{1}^{*}-\mathbf{x}_{1} e_{0}^{*}+\mathbf{x}_{2} e_{3}^{*}-\mathbf{x}_{3} e_{2}^{*} \\
\nabla J_{\mathbf{w}_{2}^{*}}= & \nabla_{\mathbf{w}_{2}^{*}}\left(e_{0} e_{0}^{*}\right)+\nabla_{\mathbf{w}_{2}^{*}}\left(e_{1} e_{1}^{*}\right) \\
& +\nabla_{\mathbf{w}_{2}^{*}}\left(e_{2} e_{2}^{*}\right)+\nabla_{\mathbf{w}_{2}^{*}}\left(e_{3} e_{3}^{*}\right) \\
= & \mathbf{x}_{0} e_{2}^{*}-\mathbf{x}_{1} e_{3}^{*}-\mathbf{x}_{2} e_{0}^{*}+\mathbf{x}_{3} e_{1}^{*} \\
\nabla J_{\mathbf{w}_{3}^{*}}= & \nabla_{\mathbf{w}_{3}^{*}}\left(e_{0} e_{0}^{*}\right)+\nabla_{\mathbf{w}_{3}^{*}}\left(e_{1} e_{1}^{*}\right) \\
& +\nabla_{\mathbf{w}_{3}^{*}}\left(e_{2} e_{2}^{*}\right)+\nabla_{\mathbf{w}_{3}^{*}}\left(e_{3} e_{3}^{*}\right) \\
= & \mathbf{x}_{0} e_{3}^{*}+\mathbf{x}_{1} e_{2}^{*}-\mathbf{x}_{2} e_{1}^{*}-\mathbf{x}_{3} e_{0}^{*} .
\end{aligned}
$$

Inserting (A.2) into (10), the derivatives of the real-valued cost function with respect to $\mathbf{w}^{\dagger}$ can be rewritten as

$$
\begin{aligned}
\nabla J_{\mathbf{w}^{\dagger}}= & \mathbf{x}_{0}\left(-e_{0}^{*}+e_{1}^{*} i+e_{2}^{*} j+e_{3}^{*} k\right) \\
& +\mathbf{x}_{1} i\left(-e_{0}^{*}+e_{1}^{*} i+e_{2}^{*} j+e_{3}^{*} k\right) \\
& +\mathbf{x}_{2} j\left(-e_{0}^{*}+e_{1}^{*} i+e_{2}^{*} j+e_{3}^{*} k\right) \\
& +\mathbf{x}_{3} k\left(-e_{0}^{*}+e_{1}^{*} i+e_{2}^{*} j+e_{3}^{*} k\right) \\
= & -\mathbf{x} e^{\dagger} .
\end{aligned}
$$

\section{Conflict of Interests}

The authors declare that there is no conflict of interests regarding the publication of this paper.

\section{Acknowledgment}

This work was supported by the National Natural Science Foundation of China under Grants 60872088 and 61172126.

\section{References}

[1] T. Bülow and G. Sommer, "Hypercomplex signals-a novel extension of the analytic signal to the multidimensional case," IEEE Transactions on Signal Processing, vol. 49, no. 11, pp. 28442852, 2001.

[2] S. Miron, N. le Bihan, and J. I. Mars, "Quaternion-MUSIC for vector-sensor array processing," IEEE Transactions on Signal Processing, vol. 54, no. 4, pp. 1218-1229, 2006.

[3] N. L. Bihan, S. Miron, and J. I. Mars, "MUSIC algorithm for vector-sensors array using biquaternions," IEEE Transactions on Signal Processing, vol. 55, no. 9, pp. 4523-4533, 2007.

[4] X. Gong, Y. Xu, and Z. Liu, "Quaternion ESPRIT for direction finding with a polarization sentive array," in Proceeding of the 9th International Conference on Signal Processing (ICSP '08), pp. 378-381, Beijing, China, October 2008.

[5] T. A. Ell and S. J. Sangwine, "Hypercomplex Fourier transforms of color images," IEEE Transactions on Image Processing, vol. 16, no. 1, pp. 22-35, 2007. 
[6] J. Seberry, K. Finlayson, S. S. Adams, T. A. Wysocki, T. Xia, and B. J. Wysocki, "The theory of quaternion orthogonal designs," IEEE Transactions on Signal Processing, vol. 56, no. 1, pp. 256265, 2008.

[7] D. Choukroun, I. Y. Bar-Itzhack, and Y. Oshman, "Novel quaternion Kalman filter," IEEE Transactions on Aerospace and Electronic Systems, vol. 42, no. 1, pp. 174-190, 2006.

[8] C. C. Took and D. P. Mandic, "The quaternion LMS algorithm for adaptive filtering of hypercomplex processes," IEEE Transactions on Signal Processing, vol. 57, no. 4, pp. 1316-1327, 2009.

[9] C. C. Took and D. P. Mandic, "Quaternion-valued stochastic gradient-based adaptive IIR filtering," IEEE Transactions on Signal Processing, vol. 58, no. 7, pp. 3895-3901, 2010.

[10] J. Vía, D. P. Palomar, L. Vielva, and I. Santamaría, "Quaternion ICA from second-order statistics," IEEE Transactions on Signal Processing, vol. 59, no. 4, pp. 1586-1600, 2011.

[11] J. Vía, D. Ramírez, and I. Santamaría, "Properness and widely linear processing of quaternion random vectors," IEEE Transactions on Information Theory, vol. 56, no. 7, pp. 3502-3515, 2010.

[12] C. C. Took and D. P. Mandic, "Augmented second-order statistics of quaternion random signals," Signal Processing, vol. 91, no. 2, pp. 214-224, 2011.

[13] C. C. Took and D. P. Mandic, "A quaternion widely linear adaptive filter," IEEE Transactions on Signal Processing, vol. 58, no. 8, pp. 4427-4431, 2010.

[14] B. C. Ujang, C. C. Took, and D. P. D. Mandic, "Quaternionvalued nonlinear adaptive filtering," IEEE Transactions on Neural Networks, vol. 22, no. 8, pp. 1193-1206, 2011.

[15] S. Javidi, C. C. Took, C. Jahanchahi, N. le Bihan, and D. P. Mandic, "Blind extraction of improper quaternion sources," in Proceeding of the IEEE International Conference on Acoustics, Speech and Signal Processing (ICASSP '11), pp. 3708-3711, Prague, Czech Republic, May 2011.

[16] R. T. Compton Jr., "On the performance of a polarization sensitive adaptive array," IEEE Transactions on Antennas and Propagation, vol. 29, no. 5, pp. 718-725, 1981.

[17] R. T. Compton Jr., "The performance of a tripole adaptive array against cross-polarized jamming," IEEE Transactions on Antennas and Propagation, vol. 31, no. 4, pp. 682-685, 1983.

[18] A. Nehorai, K.-C. Ho, and B. T. G. Tan, "Minimum-noise-variance beamformer with an electromagnetic vector sensor," IEEE Transactions on Signal Processing, vol. 47, no. 3, pp. 601-618, 1999.

[19] K. T. Wong, "Blind beamforming geolocation for widebandFFHs with unknown hop-sequences," IEEE Transactions on Aerospace and Electronic Systems, vol. 37, no. 1, pp. 65-76, 2001.

[20] J. Tao and W. Chang, "Quaternion MMSE algorithm and its application in beamforming," Acta Aeronautica et Astronautica Sinica, vol. 32, no. 4, pp. 729-738, 2011.

[21] Z.-Y. Lin, J.-W. Tao, and F. Yu, "Adaptive array filter using a combined QLMS-CLMS algorithm," in Proceeding of the 3rd IEEE International Conference on Signal Processing Systems, pp. 118121, August 2011.

[22] X. Gou, Y. Xu, Z. Liu, and X. Gong, "Quaternion-capon beamformer using crossed-dipole arrays," in Proceedng of the 4th IEEE International Symposium on Microwave, Antenna, Propagation and EMC Technologies for Wireless Communications (MAPE '11), pp. 34-37, Beijing, China, November 2011.

[23] J.-W. Tao and W.-X. Chang, "The MVDR beamformer based on hypercomplex processes," in Proceeding of the IEEE International Conference on Computer Science and Electronics Engineering (ICCSEE '12), pp. 273-277, Hangzhou, China, March 2012.
[24] J.-W. Tao, "Performance analysis for interference and noise canceller based on hypercomplex and spatiotemporal-polarisation processes," IET Radar, Sonar and Navigation, vol. 7, no. 3, pp. 277-286, 2013.

[25] J.-W. Tao and W.-X. Chang, "A novel combined beamformer based on hypercomplex processes," IEEE Transactions on Aerospace and Electronic Systems, vol. 49, no. 2, pp. 1276-1289, 2013.

[26] J. W. Tao and W. X. Chang, "The generalized sidelobe canceller based on quaternion widely linear processing," The Scientific World Journal, vol. 2014, Article ID 942923, 12 pages, 2014.

[27] H. Van Trees, Optimum Array Prosessing (Detection Estimation, and Modulation Theory), Part IV, Wiley-Interscience, New York, NY, USA, 2002.

[28] S. Said, N. le Bihan, and S. J. Sangwine, "Fast complexified quaternion Fourier transform," IEEE Transactions on Signal Processing, vol. 56, no. 4, pp. 1522-1531, 2008.

[29] Y. Tian, "Matrix theory over the complex quaternion algebra," Cornell University Library, Canada, 2000, http://arxiv.org/abs/ math/0004005.

[30] S. M. Kay, Fundamentals of Statistical Signal Processing: Estimation Theory, Prentice Hall PTR, New Jersey, NJ, USA, 1993.

[31] S. Haykin, Adaptive Filter Theoty, Prentice Hall PTR, New Jersey, NJ, USA, 4th edition, 2001.

[32] D. Bliss, A. Chan, and A. Eapen, Vector Sensor Array Design, MIT Lincon Laboratoy Presentation, Lexington, Mass, USA, 2004.

[33] H. S. Mir and J. D. Sahr, "Passive direction finding using airborne vector sensors in the presence of manifold perturbations," IEEE Transactions on Signal Processing, vol. 55, no. 1, pp. 156164, 2007.

[34] J.-J. Xiao and A. Nehorai, "Optimal polarized beampattern synthesis using a vector antenna array," IEEE Transactions on Signal Processing, vol. 57, no. 2, pp. 576-587, 2009. 


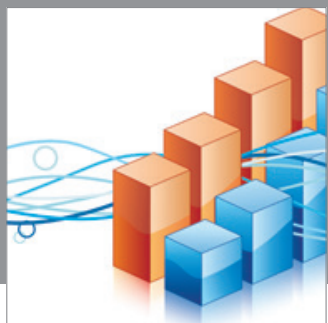

Advances in

Operations Research

mansans

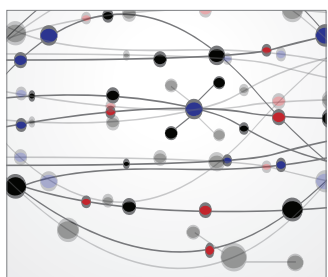

The Scientific World Journal
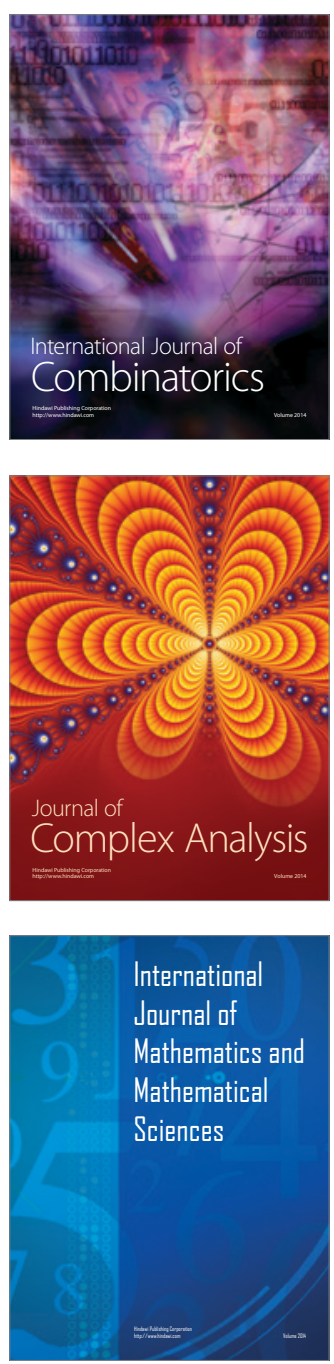
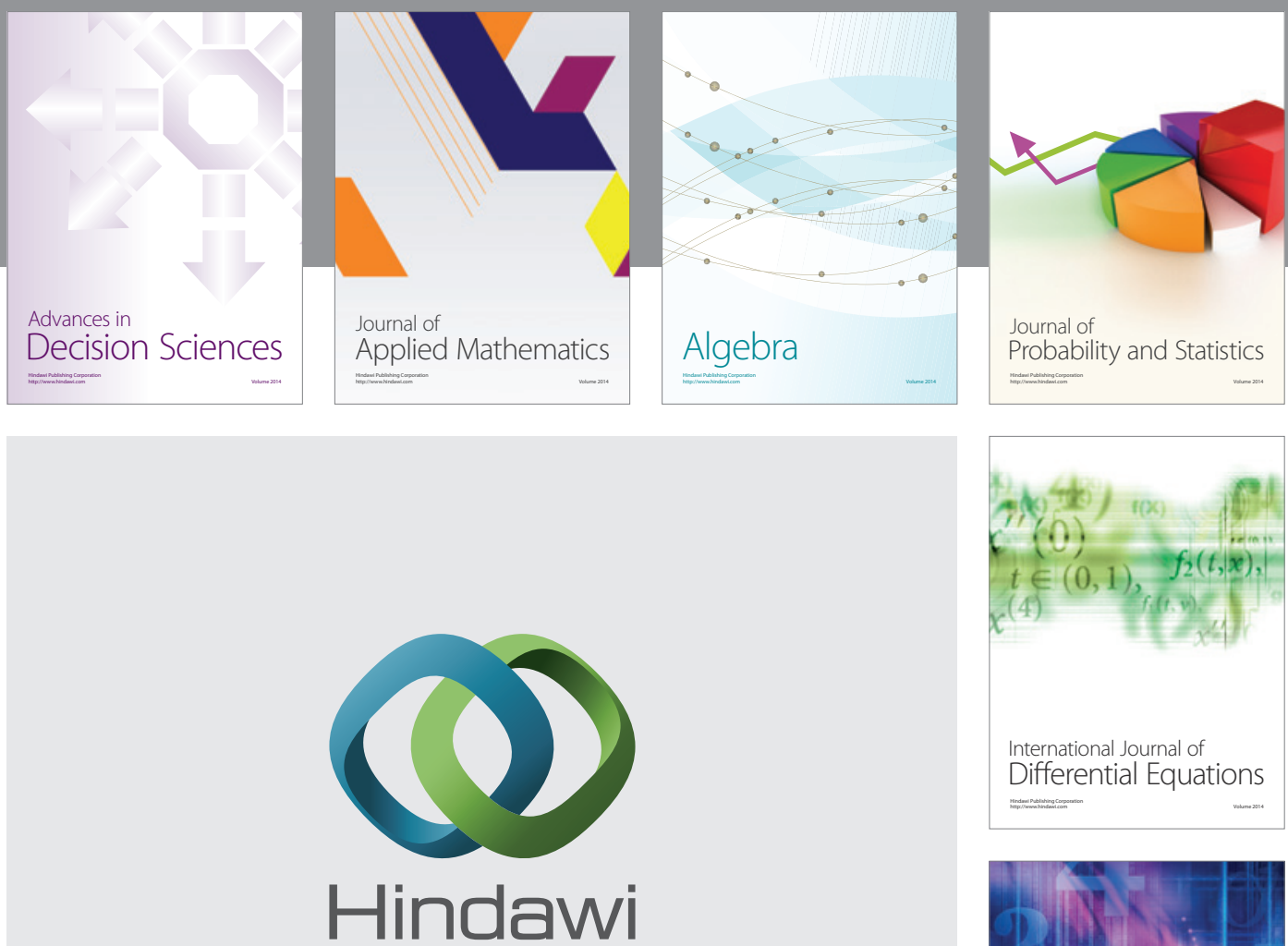

Submit your manuscripts at http://www.hindawi.com
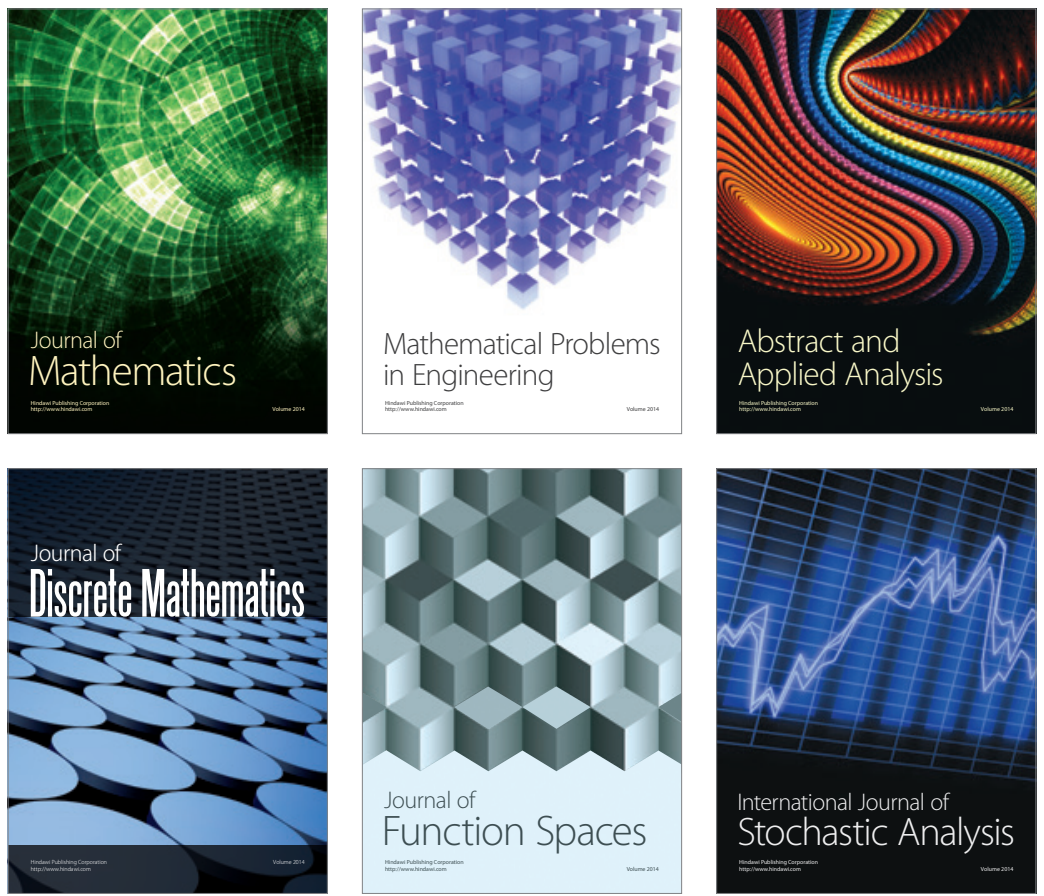

Journal of

Function Spaces

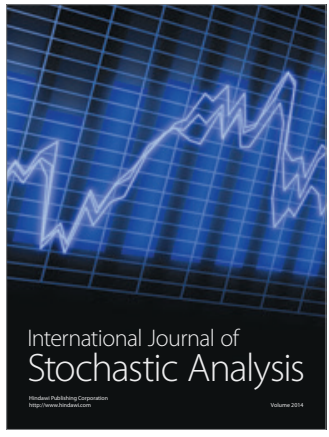

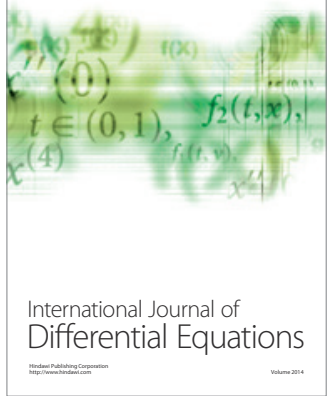
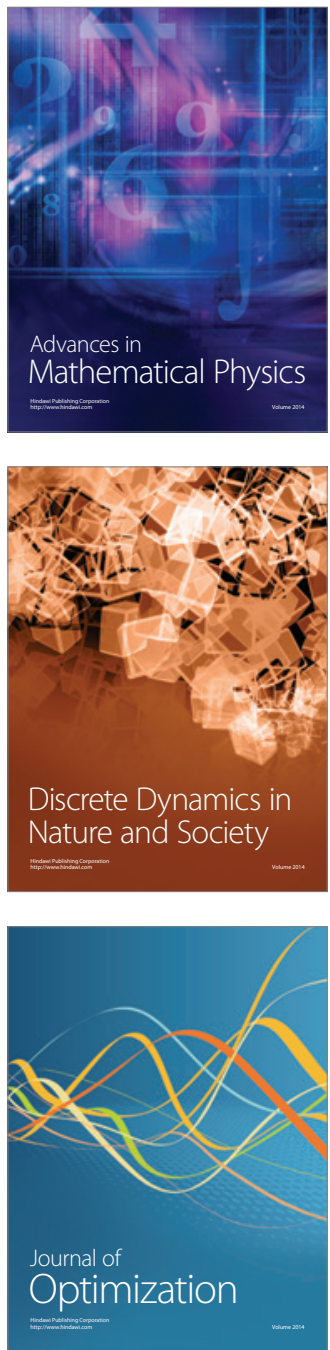